\title{
Visual outcomes after endoscopic pituitary adenomas surgery: our experience
}

\author{
Carla Fernandes ${ }^{1}$, Lídia Nunes Dias $^{2}$, Tiago Lorga ${ }^{3}$, Pedro Arede ${ }^{1}$, João Costa ${ }^{1}$ \\ ${ }^{1}$ Serviço de Oftalmologia Hospital de Egas Moniz, CHLO, Lisboa, Portugal \\ ${ }^{2}$ Serviço de Neurocirurgia Hospital de Egas Moniz, CHLO, Lisboa, Portugal \\ ${ }^{3}$ Serviço de Neurorradiologia Hospital de Egas Moniz, CHLO, Lisboa, Portugal
}

\begin{abstract}
BACKGROUND: Pituitary adenomas are the most common cause of sellar tumors after the third decade of life. They can lead to visual impairment due to a close anatomical relationship with the optic chiasm. The purpose of this study is to evaluate visual outcomes after endoscopic pituitary adenoma surgery in patients from Egas Moniz hospital between January 2013 and August 2020.

MATERIAL AND METHODS: Patients with pituitary adenoma with pre- and post-surgical ophthalmological evaluation were retrospectively included. Pre- and post-surgical visual parameters, clinical, imaging, histological, and surgical data were selected, and a descriptive analysis was performed. Pre- and post-surgical visual parameters were compared using Statistical Package for the Social Sciences (SPSS). Improvement criteria were defined: pre-surgical visual complaints > post-surgical visual complaints; visual acuity (VA) (LogMAR) pre-surgical > VA (LogMAR) post-surgical; pre-surgical chromatic vision > post-surgical chromatic vision; visual field mean sensitivity (MS) post -surgical > visual field MS pre-surgical +1 ; visual field temporal mean sensitivity (MST) post-surgical > visual field MST pre-surgical + 1; visual field nasal mean sensitivity $(\mathrm{MSN})$ post-surgical > visual field MSN pre-surgical +1 . RESULTS AND DISCUSSION: Of the total 18 patients included, 11 (68,8\%) fulfilled all improvement criteria, and 14 $(82.4 \%)$ fulfilled at least one. These results go with the current scientific evidence that pituitary adenoma resection in patients with pre-surgical visual symptoms considerably improves these symptoms.

CONCLUSION: Standardization of visual evaluation may be a key point to identify prognostic factors for visual function recovery after surgery.
\end{abstract}

KEY WORDS: pituitary adenomas; endoscopic surgery; visual function

Ophthalmol J 2021; Vol. 6, 241-244

\section{INTRODUCTION}

Pituitary adenomas account for approximately $15 \%$ of all brain tumors. They are the most common cause of sellar tumors after the third decade of life, representing $90 \%$ of all sellar masses, with a majority of prolactinomas $[1,2]$. Most of these tumors are intrasellar. However, they may extend to the suprasellar and parasellar regions and invade adjacent structures such as the cavernous sinuses and the bone of the sella turcica and clivus [3].

Clinically, they may be asymptomatic or with classic syndromes of hyper- or hypopituitarism and symptoms due to local mass effects, such as headaches, vomiting, dizziness, diplopia, or visual 
disturbance. Visual impairment is primarily due to suprasellar tumor extension with compression of the optic chiasm leading to visual field defects such as the classical bitemporal hemianopia, visual acuity or color perception reduction, and optic nerve atrophy [3]. Potential mechanisms of axonal injury from a compressive lesion include direct disruption of conduction along the axon, impaired axoplasmic flow, demyelination with impaired signal conduction, and ischemia from compression or stretching [4].

Several different visual field deficit patterns have been reported, and all relate to the position of the growing tumor relative to the optic nerves and chiasm [5]. For pituitary adenomas treatment, surgery is the gold standard, except for prolactinomas, which are mainly treated with dopamine agonists [6]. Visual field defects are recognized as one of the primary indications for surgery on pituitary tumors [2]. There are two main surgical approaches for resecting pituitary adenomas: transcranial (open) approach and endoscopic endonasal approach (EEA) [6]. Nowadays, the latter is widely accepted and adopted as proven to be a safe and effective technique in terms of tumor resection and improvement of visual defects, with reported success rates ranging from $50 \%$ to $90 \%$ [3].

The purpose of this study is to evaluate visual outcomes after endoscopic pituitary adenoma surgery in patients from Egas Moniz hospital between January 2013 and August 2020.

\section{MATERIAL AND METHODS}

This is a quantitative, observational, cross-sectional, retrospective study from Egas Moniz Hospital, Lisbon, Portugal. Authors included patients with pituitary adenoma with pre- and post-surgical ophthalmological evaluation, including visual acuity (LogMAR) and chromatic vision assessment, fundoscopy, visual field (Octopus ${ }^{\circ}$ ) testing, and optical coherence tomography (Heidelberg ${ }^{\circ}$ ) retinal nerve fibers and macular thickness evaluations (Tab. 1). Pre and post-surgical visual parameters, clinical, imaging, histological, and surgical data were selected and subjected to statistical analysis using SPSS. A descriptive analysis was performed for each parameter with measures of central location (mean/median) and dispersion (standard deviation) for quantitative variables. For qualitative variables, absolute and relative frequencies were calculated. Pre and post-surgical parameters were compared

\section{Table 1. Inclusion and exclusion criteria}

Inclusion criteria

Pituitary adenoma with pre- and post-surgical ophthalmological evaluations

Visual acuity evaluation (LogMAR)

Chromatic vison assessment

Fundoscopy evaluation

Visual field (Octopus $\left.{ }^{\circledR}\right)$ testing

Optical coherence tomography (Heidelberg ${ }^{\circledR}$ ) retinal nerve fibers and macular thickness evaluations

Exclusion criteria

Sellar tumors not pituitary adenomas

Table 2. Patient and tumor characteristics

\begin{tabular}{|l|c|}
\hline Patient and tumor characteristics & $\begin{array}{c}\text { Total population } \\
\text { (n= 18) }\end{array}$ \\
\hline Age (years) & $57.4( \pm 16.5)$ \\
\hline Male (n) & $10(55.6 \%)$ \\
\hline Average pre-surgical lesion diameter [mm] & $33.1( \pm 9.2)$ \\
\hline Aggressiveness (Ki-67) & $2.3( \pm 1.1)$ \\
\hline Pre-surgical visual complains (n) & $14(77.8 \%)$ \\
\hline $\begin{array}{l}\text { Average time of pre-surgical visual } \\
\text { complains (months) }\end{array}$ & $12.8( \pm 14.4)$ \\
\hline
\end{tabular}

using the T-test and Wilcoxon test as appropriate. A statistically significant $\mathrm{p}$-value was defined as $<0,05$.

\section{RESULTS}

Eighteen patients fulfilled our inclusion criteria. Of the 18 patients, $10(55.6 \%)$ were male, with an average age of $57.4( \pm 16.5)$ years old. Fourteen $(77.8 \%)$ patients had pre-surgical visual complaints for an average of $12.8( \pm 14.4)$ months, mostly painless and progressive vision loss $6(33.3 \%)$ and blurred vision 6 (33.3\%) (Tab. 2). Before surgery, most patients (38.9\%) showed already contact and/or opto-chiasmatic lesion, with an average lesion diameter of $33.1( \pm 9.2) \mathrm{mm}$.

The most common histological diagnosis was gonadotrophic adenoma (44.4\%), with a low mean aggressiveness (Ki-67) of $2.3( \pm 1.1)$. A statistical significance was found in the difference between the paired samples (pre- vs. post-): opto-chiasmatic relationship, right eye visual acuity, right eye visual field mean sensitivity, right eye temporal visual field mean sensitivity, left eye visual field sensitivity, left eye temporal mean sensitivity, and left eye nasal vis- 


\begin{tabular}{|c|c|c|c|}
\hline Variable & Mean Pre surgical (SD) & Mean Post surgical (SD) & p value \\
\hline Opto-chiasmatic relationship with the lesion & $2.1( \pm 1.1)$ & $1( \pm 1.5)$ & 0.047 \\
\hline VA RE (LogMAR) & $0.350( \pm 0.48)$ & $0.056( \pm 0.09)$ & 0.027 \\
\hline VA LE (LogMAR) & $0.375( \pm 0.52)$ & $0.169( \pm 0.27)$ & 0.080 \\
\hline VFMS RE & $15.7( \pm 8.1)$ & $22.5( \pm 6.3)$ & 0.010 \\
\hline VFMST RE & $10.9( \pm 9.9)$ & $20.1( \pm 8.1)$ & 0.005 \\
\hline VFMSN RE & $20.0( \pm 7.5)$ & $23.9( \pm 5.8)$ & 0.106 \\
\hline VFMS LE & $16.9( \pm 8.7)$ & $21.7( \pm 6.3)$ & 0.021 \\
\hline VFMST LE & $13.8( \pm 10.6)$ & $19.1( \pm 8.4)$ & 0.034 \\
\hline VFMSN LE & $19.8( \pm 7.9)$ & $23.8( \pm 5.0)$ & 0.038 \\
\hline MCTN RE & $601.6( \pm 44.4)$ & $593.9( \pm 49.0)$ & 0.346 \\
\hline MCTT RE & $591.5( \pm 38.4)$ & $592.4( \pm 39.9)$ & 0.650 \\
\hline MCTN LE & $598.1( \pm 45.5)$ & $594.8( \pm 51.0)$ & 0.327 \\
\hline MCTT LE & $586.3( \pm 39.4)$ & $587.8( \pm 43.9)$ & 0.702 \\
\hline RNFL RE & $79.0( \pm 14.1)$ & $79.2( \pm 15.9)$ & 0.964 \\
\hline RNFLT RE & $48.6( \pm 12.4)$ & $48.4( \pm 14.7)$ & 0.947 \\
\hline RNFLN RE & $54.5( \pm 13.2)$ & $53.8( \pm 18.2)$ & 0.830 \\
\hline RNFL LE & $75.0( \pm 14.5)$ & $75.8( \pm 19.6)$ & 0.854 \\
\hline RNFLT LE & $47.7( \pm 16.8)$ & $47.3( \pm 18.7)$ & 0.854 \\
\hline RNFLN LE & $49.7( \pm 13.0)$ & $51.9( \pm 20.5)$ & 0.559 \\
\hline
\end{tabular}

SD — standard deviation; VA — visual acuity; RE — right eye; LE — left eye; VF — visual field; MS — mean sensitivity; T — temporal; N — nasal; MCT — macular central thickness; RNFL — retinal nerve fiber layer

\footnotetext{
- Pre-surgical visual complaints $>$ Post-surgical visual complaints

- VA (LogMAR) pre-surgical > VA (LogMAR) post-surgical

- Pre-surgical chromatic vision > Post-surgical chromatic vision

- Visual field MS post-surgical > Visual field MS pre-surgical + 1

- Visual field MST post-surgical > Visual field MST pre-surgical +1

- Visual field MSN post-surgical > Visual field MSN pre-surgical +1
}

FIGURE 1. Visual improvement criteria

ual field mean sensitivity (Tab. 3). Concerning improvement in tested variables (Fig. 1), 11 (68.8\%) patients improved in all variables, and $14(82.4 \%)$ improved in at least one (Tab. 4).

\section{DISCUSSION}

Unfortunately, our sample did not have statistical strength to establish correlations between the clinical, imaging, histological, and ophthalmological evaluated criteria and the probability of improvement after surgery.

\begin{tabular}{|l|c|c|}
\hline \multicolumn{3}{|l|}{ Table 4. Visual improvement } \\
\hline At least one criteria & $14(82,4)$ & $17(100)$ \\
\hline In all criteria & $11(68,8)$ & $17(100)$ \\
\hline
\end{tabular}

Following resection of pituitary adenomas in patients with baseline visual field deficits, visual fields appear to recover in 3 stages:

- rapid recovery (minutes to days);

- delayed recovery (weeks to months);

- late recovery (months to years).

Rapid recovery was attributed to alleviating the conducting blockade, while delayed/late recovery was due to remyelination and restoration of axonoplasmic transportation, resulting in a recovery of the retinal ganglion cells [5].

According to current scientific evidence, patients with preoperative visual symptoms considerably improve after surgery. A 2017 systematic review showed that of $18-84 \%$ patients with pituitary adenoma with pre-surgical visual complaints, $67.5 \%$ improved visual acuity and $80.8 \%$ in the visual field after surgery [7]. 
The major limitation of our study is the small number of patients and some data missing that disabled us from establishing prognostic factors, as some studies advocate. In a retrospective study of 73 patients, Barzaghi et al. noted that the significant factors for complete recovery of the visual field after pituitary adenoma surgery included lower visual field mean defect before surgery, younger age, and small tumor diameter. The same study indicates that neither gender nor the symptom onset-surgery time was associated with postoperative prognosis [2]. Other authors highlight duration of symptoms, age, visual deficit, retinal nerve fiber layer thickness, and surgeon experience as significant prognostic factors for complete recovery after surgery $[3,5-7]$.

\section{CONCLUSION}

Our results are in concordance with the reported literature. A prospective study with standardization of visual evaluation could provide information on postoperative prognosis.

\section{REFERENCES}

1. Snyder R, Fayed I, Dowlati E, et al. Pituitary Adenoma and Craniopharyngioma Collision Tumor: Diagnostic, Treatment Considerations, and Review of the Literature. World Neurosurg. 2019; 121: 211-216, doi: 10.1016/..wneu.2018.10.048, indexed in Pubmed: 30326307.

2. Lee DK, Sung MiS, Park SW. Factors Influencing Visual Field Recovery after Transsphenoidal Resection of a Pituitary Adenoma. Korean $\mathrm{J}$ Ophthalmol. 2018; 32(6): 488-496, doi: 10.3341/kjo.2017.0094, indexed in Pubmed: 30549473.

3. Fredes $F$, Undurraga G, Rojas $P$, et al. Visual Outcomes after Endoscopic Pituitary Surgery in Patients Presenting with Preoperative Visual Deficits. J Neurol Surg B Skull Base. 2017; 78(6): 461-465, doi: 10.1055/s-0037-1604169, indexed in Pubmed: 29134164.

4. Kerrison JB, Lynn MJ, Baer CA, et al. Stages of improvement in visual fields after pituitary tumor resection. Am J Ophthalmol. 2000; 130(6): 813-820, doi: 10.1016/s0002-9394(00)00539-0, indexed in Pubmed: 11124302.

5. Uy B, Wilson B, Kim WiJ, et al. Visual Outcomes After Pituitary Surgery. Neurosurg Clin N Am. 2019; 30(4): 483-489, doi: 10.1016/j. nec.2019.06.002, indexed in Pubmed: 31471055.

6. Shen M, Chen Z, Shou X, et al. Surgical Outcomes and Predictors of Visual Function Alterations After Transcranial Surgery for Large-toGiant Pituitary Adenomas. World Neurosurg. 2020; 141: e60-e69, doi: 10.1016/j.wneu.2020.04.151, indexed in Pubmed: 32353541.

7. Muskens IS, Zamanipoor Najafabadi AH, Briceno V, et al. Visual outcomes after endoscopic endonasal pituitary adenoma resection: a systematic review and meta-analysis. Pituitary. 2017; 20(5): 539-552, doi: 10.1007/s11102-017-0815-9, indexed in Pubmed: 28643208. 